\title{
Alternativas de tratamiento para la osteonecrosis de los maxilares asociada a bisfosfonatos
}

\section{Treatment alternatives for bisphosphonate associated osteonecrosis of the jaw}

\author{
Ardila Medina $\mathrm{CM}^{*}$
}

\section{RESUMEN}

Se ha notificado recientemente un incremento de la osteonecrosis en los pacientes que reciben bisfosfonatos que contienen nitrógeno. Clínicamente, la necrosis ósea maxilar o mandibular puede ocurrir de forma espontánea o aparecer secundariamente a una exodoncia o trauma que afecte los maxilares. La necrosis se manifiesta por exposición del hueso en el sitio afectado, inflamación de los tejidos blandos adyacentes, halitosis, dolor y fiebre; puede producir fístula mucosa o cutánea. También se observa que los pacientes tratados con administración intravenosa de bisfosfonatos se encuentran en alto riesgo de desarrollar focos osteonecróticos únicos o múltiples, situación advertida esporádicamente en los sujetos tratados con administración oral. Se han indicado diferentes recomendaciones con el fin de establecer los procedimientos efectivos para tratar la osteonecrosis asociada a bisfosfonatos. Los tratamientos comprenden alternativas no quirúrgicas, antibióticos, terapia hiperbárica, cirugía convencional o mediante láser. Adicionalmente, las medidas preventivas son un aspecto importante que debe tenerse en cuenta.

Palabras clave: Bisfosfonatos, osteonecrosis, terapia.

\section{SUMMARY}

A relative increase in osteonecrosis has been reported in recent years particularly among patients receiving nitrogen-containing bisphosphonates. Clinically, mandibular or maxillary necrosis may occur spontaneously or be secondary to tooth extraction or trauma affecting the jaws. Necrosis typically manifests itself with exposure of the bone at the site involved and swelling of the adjacent soft tissues, halitosis, pain and fever, and may produce cutaneous or mucosal fistulae. It has also been observed that patients who have taken bisphosphonates by intravenous administration appear to be at a higher risk of developing single or multiple osteonecrotic foci of the jaw bones, whereas this has only been reported sporadically in patients with oral administration Several recommendations have been made to establish effective procedures to treat biphosphonate-associated osteonecrosis. The treatment ranges from nonsurgical approach to conventional surgery through medical treatment, antibiotic regimen, hyperbaric therapy and laser surgery. Beside, the importance of preventive measures is thus highlighted.

Key words: Bisphosphonates, osteonecrosis, therapy.

Fecha de recepción: 2 de abril de 2009.

Aceptado para publicación: 11de abril de 2009.

* Profesor Asistente Universidad de Antioquía. Miembro Junta Directiva Asociación Colombiana de Periodoncia y Oseointegración.

Ardila Medina CM. Alternativas de tratamiento para la osteonecrosis de los maxilares asociada a bisfosfonatos. Av. Odontoestomatol 2010; 26 (3): 153-159. 


\section{INTRODUCCIÓN}

La estructura química de los bisfosfonatos (BFs) es similar a la del pirofosfato orgánico, caracterizada por inhibir la reabsorción ósea $(1,2)$. En particular, presentan gran afinidad por la hidroxiapatita y están orientados a sitios de remodelación ósea activa. No se conoce completamente su mecanismo de acción, pero se cree que inhibe la reabsorción ósea por medio de efectos directos sobre los osteoclastos u otras células óseas $(1,3)$. Los BFs pueden agruparse químicamente en dos categorías principales: con y sin contenido de nitrógeno. Las cadenas de nitrógeno previenen la metabolización permitiendo su acumulación con efectos continuos $(1,4)$. Se administran para una gran cantidad de enfermedades metabólicas y oncológicas incluyendo osteoporosis, enfermedad de Paget, mieloma múltiple y enfermedad ósea metastásica (4). Los BFs orales se prescriben regularmente para pacientes con enfermedad de Paget y osteoporosis postmenopáusica (5), mientras que los BFs más potentes, como el pamidronato y el ácido zoledrónico se administran intravenosamente y están indicados como terapia en caso de fracturas patológicas, hipercalcemias en cáncer metastásico y para tratar defectos de reabsorción ósea en mielomas múltiples (6).

Los BFs son generalmente bien tolerados. Sus efectos adversos son poco frecuentes y consisten en altos niveles de creatinina, fatiga, artralgia, náuseas, daño en la función renal, hipocalcemia, incremento en el dolor óseo y osteonecrosis avascular de los maxilares (7); aparentemente no involucra otros huesos. Los síntomas generalmente incluyen dificultad para comer y hablar, inflamación, dolor, sangrado, parestesia del labio inferior, además de pérdida y movilidad dental (3). Los hallazgos radiográficos no son específicos y las lesiones deben ser sometidas a biopsias para descartar metástasis (8).

El objetivo de este artículo es describir los tratamientos empleados para tratar la osteonecrosis asociada a bifosfonatos (OABs), los cuales abarcan alternativas no quirúrgicas, antibióticos, terapia hiperbárica, cirugía convencional o mediante láser. También se detallarán las medidas preventivas más importantes que deben formalizarse en los pacientes que van a ser sometidos a terapias con BFs.

\section{MEDIDAS PREVENTIVAS}

Diferentes autores y asociaciones científicas médicas/odontológicas han ilustrado la importancia de la aplicación de medidas preventivas en pacientes que van a ser sometidos a medicación con BFs intravenosos (9-12). Antes de prescribir la terapia con bisfosfonatos el médico debe remitir el paciente al odontólogo para una evaluación integral inmediata. El examen dental debe incluir una evaluación clínica completa, además de radiografías panorámicas y periapicales. El tratamiento odontológico ayuda a eliminar las infecciones y a prevenir la necesidad de procedimientos dentales invasivos en el futuro inmediato. Las intervenciones pueden abarcar exodoncias, cirugía periodontal, terapia endodóntica, control de caries, restauraciones y prótesis; una vez realizado alguno de estos procedimientos, se debe esperar un mes de cicatrización antes de iniciar la prescripción de BFs. Algunos autores indican que estos pacientes no deben ser candidatos para restaurar con implantes ya que presentan predisposición a exposición ósea (13), sin embargo, otros investigadores sugieren que siguiendo algunas consideraciones especiales tal opción de tratamiento es factible $(14,15)$. No deben intervenirse quirúrgicamente los dientes impactados que se encuentren completamente cubiertos por hueso o tejidos blandos, pero si deben removerse aquellos dientes que presenten comunicaciones orales, esperando también, como mínimo, un mes de cicatrización previo al comienzo de la medicación con BFs. Se recomiendan evaluaciones odontológicas periódicas cuando comience la terapia con BFs. Un estudio prospectivo encontró que el riesgo de OABs disminuye después de la implementación de medidas dentales preventivas (16). Los investigadores estudiaron si la presencia de OABs en 128 pacientes disminuía con la aplicación de medidas preventivas en pacientes que recibían ácido zoledrónico. Los sujetos fueron estratificados en dos grupos $A(n=38)$ y $B(n=90)$ si el tratamiento iniciaba antes o después de la aplicación de las medidas preventivas. El 12,5\% (16 sujetos) de los pacientes desarrollaron OABs; ocho del grupo A $(26,3 \%$ ) y dos del grupo B $(6,7 \%)$ $(p=0,002)$. La tasa de incidencia (TI) para el grupo $A$ fue de $0,671 / 100$ persona-mes y para el grupo B $0,230 / 100$ persona-mes (razón TI 2,92, $p=0,029$, IC $95 \%$ 1,06-8,03). Ningún paciente del grupo B pre- 
sentó estado III de OABs. Los resultados de este estudio son corroborados por Ripamonti y colaboradores (17), quienes investigaron la presencia de OABs antes y después de la aplicación de medidas preventivas al iniciar la terapia con BFs. Realizaron una evaluación oral (visita odontológica \pm ortopantomografía de los maxilares) en 154 sujetos (grupo post) con el fin de detectar condiciones dentales potenciales y la necesidad de tratamiento odontológico. Igualmente, efectuaron una revisión retrospectiva de pacientes con cáncer y metástasis ósea (grupo pre), tratados por primera vez con BFs, desde 1999 hasta 2005 sin recibir ninguna medida preventiva. Se observó osteonecrosis de los maxilares en 28 pacientes (2,9\%); se presentó una reducción en la incidencia de osteonecrosis de 3,2\% a 1,3\%, al comparar la aplicación de medidas preventivas en los grupos pre y post. Considerando los pacientes expuestos al ácido zoledrónico, la realización de un examen dental y la aplicación de medidas preventivas, llevó a una reducción sostenida de la TI de osteonecrosis $(7,8 \%$ en el grupo pre y $1,7 \%$ en el grupo post; $p=0,016$ ). Los autores concluyeron que la osteonecrosis de los maxilares es una condición que se puede manejar y prevenir. Adicionalmente, los exámenes odontológicos combinados con la identificación de pacientes en riesgo pueden mejorar los resultados y disminuir el número de pacientes con osteonecrosis.

\section{ALTERNATIVAS DE TRATAMIENTO}

La OABs orales es habitualmente menos extensa y responde mejor al tratamiento cuando se compara con la osteonecrosis de los maxilares asociadas a BFs intravenosos $(9,13)$. Marx y colaboradores (13), realizaron un estudio con el fin de contrastar la información obtenida a partir del seguimiento de 30 casos de OABs orales con 116 casos inducidos por BFs intravenosos, relacionando factores de riesgo y opciones de tratamiento. Los autores encontraron menor frecuencia, más predecibilidad y mejor respuesta al tratamiento en pacientes con OABs orales; recomiendan al clínico contactar al médico tratante para informar la complicación, en aquellos pacientes que presentan exposiciones óseas debidas a BFs. Cuando la exposición ósea es asintomática se sugiere mantenimiento con clorhexidina al $0,12 \%$. Se recomienda terapia antibiótica adicional al enjuague con clorhexidina si el paciente relata dolor y/o existe evidencia clínica de infección (9-13). El antibiótico de elección es penicilina V-K, en dosis de 500 mg cada seis horas debido a su eficacia contra las cepas más comunes asociadas a infecciones secundarias por exposición ósea a BFs (actinomyces, eikenella y moraxella) (13). Si el paciente es alérgico a la penicilina se puede prescribir doxiciclina (100 $\mathrm{mg} /$ día), levofloxacina (500 mg/día) o azitromicina (250 mg/día). El régimen antibiótico debe extenderse durante 14 días o hasta que exista remisión del dolor. En casos muy sintomáticos o refractarios el metronidazol (500 mg 3 veces/día) es de gran utilidad. Algunos autores consideran que gran parte de los casos de OABs orales se resuelven sin necesidad de cirugía durante el período en el que se suspenden los BFs, sin embargo, indican que se puede realizar un debridamiento local si existe movilidad ósea o evidencia radiográfica de secuestros óseos (13).

La terapia conservadora también ha sido recomendada en los casos de OABs intravenosos $(18,19)$. Hoff y colaboradores (18), realizaron un análisis retrospectivo en 4019 pacientes tratados con BFs intravenosos, entre 1996 y 2004. La dosis media y la duración del tratamiento con pamidronato y ácido zoledrónico fueron significativamente superiores en pacientes con osteonecrosis de los maxilares ( $p<0,0001)$. El análisis multivariado de riesgos proporcionales de Cox identificó a las exodoncias (hazards ratio [HR], 53,19; intervalo de confianza (IC) del 95\%: 18,20-155,46; $\mathrm{p}<0,0001$ ) como un factor de riesgo significativo para osteonecrosis. Trece de 29 pacientes fueron seguidos durante un período promedio de 17 meses; las lesiones cicatrizaron en tres pacientes durante ese período, encontrando mejoría en el 23\% de los pacientes manejados con terapia conservadora. Van den Wyngaert y colaboradores (19), evaluaron un tratamiento conservador (clorhexidina al $0,12 \%$, antibióticos intermitentes y secuestromía moderada) en 33 pacientes con OABs. Durante un período de seguimiento de 23 meses, el $53 \%$ de los sujetos no presentó exposición ósea, $37 \%$ manifestó lesiones estables y el 10\% restante mostró necrosis progresiva. La suspensión de los Bs no influyó en el resultado. Los autores demostraron que el tratamiento conservador produjo el cierre de la mucosa en el $53 \%$ de los pacientes. 
A pesar de los resultados obtenidos por la terapia conservadora, el tratamiento quirúrgico está indicado en algunos casos. Wutzl y colaboradores (20), realizaron el primer estudio prospectivo que evaluó el resultado del tratamiento quirúrgico después de seis meses, en una cohorte de 58 pacientes con osteonecrosis de los maxilares. Las variables resultado evaluadas fueron: estado de la mucosa, dolor y rehabilitación protésica. Después de la terapia quirúrgica el $58.5 \%$ de los pacientes estuvieron libres de dolor y presentaron una mucosa intacta; once de 12 pacientes tratados con procedimientos a colgajo para cierre de tejidos blandos presentaron una mucosa sana. Los autores demostraron que una estrategia de tratamiento posible en pacientes con osteonecrosis se puede conseguir mediante una resección mínima del hueso necrótico y cierre local de los tejidos blandos. Longobardi y colaboradores (21), evaluaron 18 pacientes afectados por OABs. La mayoría de las lesiones tratadas se encontraron en áreas edéntulas, en pacientes con prótesis dentales desajustadas y en sitios tratados con implantes o periodontitis. Después de la anamnesis y la valoración clínica de los pacientes, se realizó terapia profesional en higiene oral y se indicaron enjuagues con clorhexidina al 0,2\% tres veces al día. Después de estas recomendaciones, los pacientes fueron intervenidos quirúrgicamente. El procedimiento consistió en la remoción de los márgenes de la mucosa ulcerada y del borde óseo hasta encontrar un hueso compacto cubierto por periostio. Se administró amoxicilina/clavulánico por vía intravenosa, cada 6 horas durante seis días y se indicó continuar con los enjuagues de clorhexidina durante un mes. Se efectuaron controles semanales durante el primer mes y luego cada tres semanas hasta el sexto mes. Tres pacientes murieron debido a sus complicaciones oncológicas, cuatro mostraron resolución de sus lesiones, seis presentaron mejoría de las mismas y en siete se observaron alivio de los síntomas sin cambio en la lesión. Todos los pacientes refirieron sensación de boca fresca y desaparición del olor fétido.

Diferentes estudios han notificado el empleo de láser en el tratamiento de la OABs $(22,23)$. La mayoría de estudios sobre bioestimulación ósea han mostrado la efectividad de los láseres visible, HeNe (helio/ neón) y diodo infrarrojo en diversas longitudes de onda $(24,25)$. Angiero y colaboradores (22), evalua- ron en un estudio retrospectivo el uso del láser Er:YAG en pacientes diagnosticados con osteonecrosis y tratados con BFs de última generación. Se identificaron 49 pacientes con osteonecrosis mandibular; 19 fueron tratados con terapia conservadora, 20 con cirugía radical y 10 con láser Er:YAG. Algunos de los pacientes tratados quirúrgicamente requirieron retratamiento, terminando en fracturas óseas. Los sujetos tratados conservadoramente mejoraron los síntomas pero no presentaron remisión de las lesiones. De los tratados con láser, seis lograron remisión total de los signos y síntomas, cuatro presentaron mejoría y un caso requirió retratamiento. Los autores concluyeron que el tratamiento con láser condujo a mejoría significativa en los parámetros clínicos, representando una alternativa válida para el tratamiento de osteonecrosis por BFs. Igualmente, Vescovi y colaboradores (23), evaluaron los resultados de tratamiento con láser, en este caso el Nd:Yag. Un total de 28 sujetos con OABs fueron divididos en cuatro grupos según el tipo de tratamiento: ocho tratados con terapia médica solamente (antibióticos con/sin antimicóticos y/o enjuagues antisépticos), seis con terapia médica y quirúrgica (remoción de restos óseos necróticos y curetaje óseo), seis tratados con terapia médica asociada a bioestimulación con láser, y ocho manejados con antibióticos asociados a terapia quirúrgica y láser. De los 14 pacientes intervenidos con láser nueve presentaron completo éxito clínico y dos mejoraron su sintomatología. Dos sujetos no presentaron mejoría clínica y uno presentó recurrencia de osteonecrosis en el área tratada.

La terapia con oxígeno hiperbárico $(\mathrm{OH})$ también se ha considerado como una opción viable para el tratamiento de OABs. La racionalización de su uso terapéutico está determinada debido a que la apoptosis osteoclástica promovida por el nitrógeno de los BFs puede suprimirse mediante citoquinas osteoclastogénicas; estas señales son conocidas por su sensibilidad al oxígeno (26). Freiberger y colaboradores (27), realizaron un estudio piloto en 16 pacientes con el fin de evaluar el posible papel del $\mathrm{OH}$ en el tratamiento de pacientes con OABs. Cuarenta sesiones de $\mathrm{OH}$ condujeron a la remisión de la sintomatología o al mejoramiento del $62,5 \%$ de los pacientes. El $44 \%$ de los pacientes remitieron los síntomas inmediatamente después de la terapia y el $50 \%$ lograron la estabilización de los mismos, sin 
embargo, se logró la estabilización sin remisión en solamente dos pacientes. Se presentó un tiempo de fracaso más corto en la respuesta al tratamiento de siete pacientes que continuaron con el uso de BFs durante la terapia con $\mathrm{OH}$ (8,5 meses; IC 95\% $=7,1-9,8)$, comparado con aquellos que descontinuaron el medicamento (20,1 meses; IC 95\% = $17,5-23,9 ; p=0,006$, log-rank test). Los autores concluyeron que la terapia adjunta con $\mathrm{OH}$ puede beneficiar los pacientes con OABs, teniendo presente que su mejoría se incrementa cuando se suspende la medicación con BFs. Estos resultados son corroborados por otros autores que han evaluado la respuesta al $\mathrm{OH}$ en un número menor de sujetos $(28,29)$. En la tabla 1 se presentan las alternativas de tratamiento sugeridas en OABs.

Existe controversia en la literatura en cuanto a la interrupción o no de la terapia con BFs durante el manejo de pacientes con OABs. Algunos autores consideran que la suspensión de la medicación con BFs no ayuda en la mejoría de los pacientes que padecen OABs debido a que sus niveles pueden persistir en el hueso durante 12 años después de

\begin{tabular}{|c|c|}
\hline \multicolumn{2}{|c|}{ TABLA 1.- ALTERNATIVAS DE } \\
TRATAMIENTO PARA LA \\
OSTEONECROSIS DE LOS MAXILARES \\
ASOCIADA A BISFOSFONATOS
\end{tabular}

terminado el tratamiento $(1,19)$. Además, la interrupción de su formulación alteraría el curso de la enfermedad ocasionando recurrencia del dolor óseo y progreso de la metástasis o de las lesiones osteolíticas (30). Sin embargo, un estudio multicéntrico realizado en 60 pacientes intervenidos con diferentes protocolos de tratamiento presentó resultados favorables en aquellos pacientes cuyo protocolo incluyó la suspensión de los BFs (31). Los autores encontraron que la interrupción de la terapia con BFs por más de seis meses seguida por debridamiento del defecto y administración de antibióticos ocasionó completa cicatrización de las heridas en siete pacientes, mientras que la suspensión de los BFs sin ningún procedimiento invasivo produjo estabilización del defecto.

\section{CONCLUSIONES}

Es imprescindible que médicos y odontólogos indiquen las medidas preventivas que deben incluirse en la evaluación integral del estado bucal y dental de los pacientes, antes de iniciar la terapia con BFs. Se debe realizar un estrecho monitoreo de la higiene oral, diagnóstico temprano y prevención de consecuencias potencialmente devastadoras. Es importante destacar la necesidad de efectuar investigación especialmente relacionada con la epidemiología de los factores de riesgo, y la fisiopatología de la osteonecrosis del maxilar y la mandíbula asociada a la medicación con BFs. Además, algunos aspectos concernientes con el tratamiento de OABs, como la suspensión o no de los BFs, la terapia con $\mathrm{OH}$ y láser, carecen de suficiente evidencia, dificultándole al clínico tomar decisiones acertadas. Esto implica llevar a cabo ensayos clínicos controlados enfocados a visualizar la terapia adecuada, con el fin de asegurar el protocolo de tratamiento propicio para el paciente.

\section{BIBLIOGRAFÍA}

1. Bagan J, Scully C, Sabater V, Jimenez Y. Osteonecrosis of the jaws in patients treated with intravenous bisphosphonates (BRONJ): A concise update. Oral Oncol 2009 Feb 27. [Epub ahead of print]. 
2. Shu G, Yamamoto K, Nagashima M. Differences in osteoclast formation between proximal and distal tibial osteoporosis in rats with adjuvant arthritis: inhibitory effects of bisphosphonates on osteoclasts. Mod Rheumatol 2006;16:343-9.

3. Silverman SL, Landesberg R. Osteonecrosis of the jaw and the role of bisphosphonates: a critical review. Am J Med 2009; 122 (2 Suppl): S33-45.

4. Farrugia MC, Summerlin D-J, Krowiak E, Huntley T, Freeman, S, Borrowdale R, et al. Osteonecrosis of the mandible or maxilla associated with the use of new generation bisphosphonates. Laryngoscope 2006;116:115-20.

5. American College of Obstetricians and Gynecologists, Women's Health Care Physicians. ACOG practice bulletin. Clinical management guidelines for obstetricians-gynecologists. Obstet Gynecol 2004;103:203-16.

6. Cetiner S, Sucak GT, Kahraman SA, Aký SZ, Kocakahyaoglu B, Gultekin SE, et al. Osteonecrosis of the jaw in patients with multiple myeloma treated with zoledronic acid. J Bone Miner Metab 2009 Feb 26. [Epub ahead of print].

7. Escobar López EA, López López J, Marques Soares MS, Chimenos Küstner E. Osteonecrosis de los maxilares asociada a bifosfonatos: revisión sistemática. Av. Odontoestomatol 2007;23: 91-101.

8. Rizzoli R, Burlet N, Cahall D, Delmas PD, Eriksen EF, Felsenberg D, et al. Osteonecrosis of the jaw and bisphosphonate treatment for osteoporosis. Bone 2008;42:841-7.

9. Edwards BJ,Hellstein JW, Jacobsen PL, Kaltman S, Mariotti A, Migliorati CA. Updated recommendations for managing the care of patients receiving oral bisphosphonate therapy. An advisory statement from the American Dental Association Council on Scientific Affairs. JADA 2008; 139:1674-77.

10. Khan AA, Sándor GK, Dore E, Morrison AD, Alsahli M, Amin F, et al. Canadian consensus practice guidelines for bisphosphonate associated osteonecrosis of the jaw. J Rheumatol 2008;35: 1391-7.

11. Campisi G, Fedele S, Colella G, Casto AL, Fusco V. Canadian consensus practice guidelines for bisphosphonate associated osteonecrosis of the jaw. J Rheumatol 2009;36:451-3.

12. Sambrook PN. Consensus practice guidelines for bisphosphonate-associated osteonecrosis of the jaw. Nat Clin Pract Rheumatol 2009;5:6-7.

13. Marx RE, Cillo JE Jr, Ulloa JJ. Oral bisphosphonate-induced osteonecrosis: risk factors, prediction of risk using serum CTX testing, prevention, and treatment. J Oral Maxillofac Surg 2007;65:2397-410.

14. Kasai T, Pogrel MA, Hossaini M. The prognosis for dental implants placed in patients taking oral bisphosphonates. J Calif Dent Assoc 2009;37:3942.

15. Aspenberg P. Bisphosphonates and implants: an overview. Acta Orthop 2009;80:119-23.

16. Dimopoulos MA, Kastritis E, Bamia C, Melakopoulos I, Gika D, Roussou M, et al. Reduction of osteonecrosis of the jaw (ONJ) after implementation of preventive measures in patients with multiple myeloma treated with zoledronic acid. Ann Oncol 2009;20:117-20.

17. Ripamonti CI, Maniezzo M, Campa T, Fagnoni E, Brunelli C, Saibene G, et al. Decreased occurrence of osteonecrosis of the jaw after implementation of dental preventive measures in solid tumour patients with bone metastases treated with bisphosphonates. The experience of the National Cancer Institute of Milan. Ann Oncol 2009;20:137-45.

18. Hoff AO, Toth BB, Altundag K, Johnson MM, Warneke CL, Hu M, et al. Frequency and risk factors associated with osteonecrosis of the jaw in cancer patients treated with intravenous bisphosphonates. J Bone Miner Res 2008;23: 826-36. 
19. Van den Wyngaert T, Claeys T, Huizing MT, Vermorken JB, Fossion E. Initial experience with conservative treatment in cancer patients with osteonecrosis of the jaw (ONJ) and predictors of outcome. Ann Oncol 2009;20:331-6.

20. Wutzl A, Biedermann E, Wanschitz F, Seemann R, Klug C, Baumann A, et al. Treatment results of bisphosphonate-related osteonecrosis of the jaws. Head Neck 2008; 30: 1224-30.

21. Longobardi G, Boniello R, Gasparini G, Pagano I, Pelo S. Surgical Therapy for osteonecrotic lesions of the Jaws in patients in therapy with bisphosphonates. J Craniofac Surg 2007;18: 1012-7.

22. Angiero F, Sannino C, Borloni R, Crippa R, Benedicenti S, Romanos GE. Osteonecrosis of the jaws caused by bisphosphonates: evaluation of a new therapeutic approach using the Er:YAG laser. Lasers Med Sci 2009 Mar 11. [Epub ahead of print]

23. Vescovi P, Merigo E, Manfredi M, Meleti M, Fornaini $C$, Bonanini $M$, et al. Nd:YAG laser biostimulation in the treatment of bisphosphonate-associated osteonecrosis of the jaw: clinical experience in 28 cases. Photomed Laser Surg 2008;26:37-46.

24. Nissan J, Assif D, Gross MD, Yaffe A, Binderman I. Effect of low intensity laser irradiation on surgically created bony defects in rats. $\mathrm{J}$ Oral Rehabil 2006;33:619-24.

25. Muniz Renno AC, Mendes de Moura F, Andrade dos Santos NS, Passarelli Tirico R, Bossini PS, Parizotto NA. Effects of infrared-830 nm laser used in two doses, on biochemical properties on osteopenic rats femora. Photomed Laser Surg 2006;24:202-6.

26. Lee NK, Choi YG, Baik JY, Han SY, Jeong DW, Bae YS, et al. A crucial role for reactive oxygen species in RANKL-induced osteoclast differentiation. Blood 2005;106:852-9.

27. Freiberger JJ, Padilla-Burgos R, Chhoeu AH, Kraft $\mathrm{KH}$, Boneta O, Moon RE, et al. Hyperbaric oxygen treatment and bisphosphonate-induced osteonecrosis of the jaw: a case series. J Oral Maxillofac Surg 2007;65:1321-7.

28. Shimura K, Shimazaki C, Taniguchi K, Akamatsu S, Okamoto M, Uchida R, et al. Hyperbaric oxygen in addition to antibiotic therapy is effective for bisphosphonate-induced osteonecrosis of the jaw in a patient with multiple myeloma. Int $\mathrm{J}$ Hematol 2006;84:343-5.

29. Mignogna MD, Fedele S, Lo Russo L, Ciccarelli R, Lo Muzio L. Case 2: Osteonecrosis of the jaws associated with bisphosphonate therapy. J Clin Oncol 2006;24:1475-7.

30. Gallego L, Junquera L. Consequence of therapy discontinuation in bisphosphonate-associated osteonecrosis of the jaws. Br J Oral Maxillofac Surg 2009;47:67-8.

31. Magopoulos C, Karakinaris G, Telioudis Z, Vahtsevanos K, Dimitrakopoulos I, Antoniadis $\mathrm{K}$, et al. Osteonecrosis of the jaws due to bisphosphonate use. A review of 60 cases and treatment proposals. Am J Otolaryngol 2007; 28 : 158-63.

\section{CORRESPONDENCIA}

Carlos Martín Ardila Medina

Carrera 47 No. 20 sur 46

Envigado Antioquía Colombia

57(4) 3348122

martinardila@gmail.com

cmartin@odontologia.udea.edu.co 